\title{
Correction to: Epigenetic priming sensitizes gastric cancer cells to irinotecan and cisplatin by restoring multiple pathways
}

\author{
Hiroshi Moro ${ }^{1,2} \cdot$ Naoko Hattori $^{1} \cdot$ Yoshiaki Nakamura ${ }^{1,3} \cdot$ Kana Kimura $^{1} \cdot$ Toshio Imai $^{4} \cdot$ Masahiro Maeda $^{1}$. \\ Masakazu Yashiro ${ }^{5} \cdot$ Toshikazu Ushijima $^{1,2}$ (1)
}

Published online: 15 November 2019

(c) The International Gastric Cancer Association and The Japanese Gastric Cancer Association 2019

\section{Correction to: Gastric Cancer \\ https://doi.org/10.1007/s10120-019-01010-1}

We recently published the priming effect of a DNA demethylating agent in chemotherapy of gastric cancer (original article). Unfortunately, there are errors in the units of some panels in Fig. 2. The units for SN38 (the most upper three panels) and PTX (the second bottom two panels) should be "nM", instead of " $\mu \mathrm{M}$ ".

The errors were produced when the unit for 5-FU (the two bottom panels) was corrected from " $\mathrm{nM}$ " to " $\mu \mathrm{M}$ " during the production stage. Accidentally, the units for SN38 and PTX were also changed. A correct Fig. 2 is shown below.

The publisher sincerely apologize for the error.

The original article can be found online at https://doi.org/10.1007/ s10120-019-01010-1.

Toshikazu Ushijima

tushijim@ncc.go.jp

1 Division of Epigenomics, National Cancer Center Research Institute, Tokyo, Japan

2 Course of Advanced Clinical Research of Cancer, Juntendo University Graduate School of Medicine, Tokyo, Japan

3 Department of Gastroenterology and Gastrointestinal Oncology, National Cancer Center Hospital East, Chiba, Japan

4 Central Animal Division, National Cancer Center Research Institute, 5-1-1 Tsukiji, Chuo-ku, Tokyo 104-0045, Japan

5 Department of Surgical Oncology, Osaka City University Graduate School of Medicine, Osaka, Japan 
Fig. 2 Effect of epigenetic priming by 5 -aza-dC. Resistant cells were first treated with 5-aza-dC or mock first, and subsequently treated with a cytotoxic drug. The $\mathrm{IC}_{50}$ values were significantly lower for SN38 and CDDP, but not for PTX and 5-FU

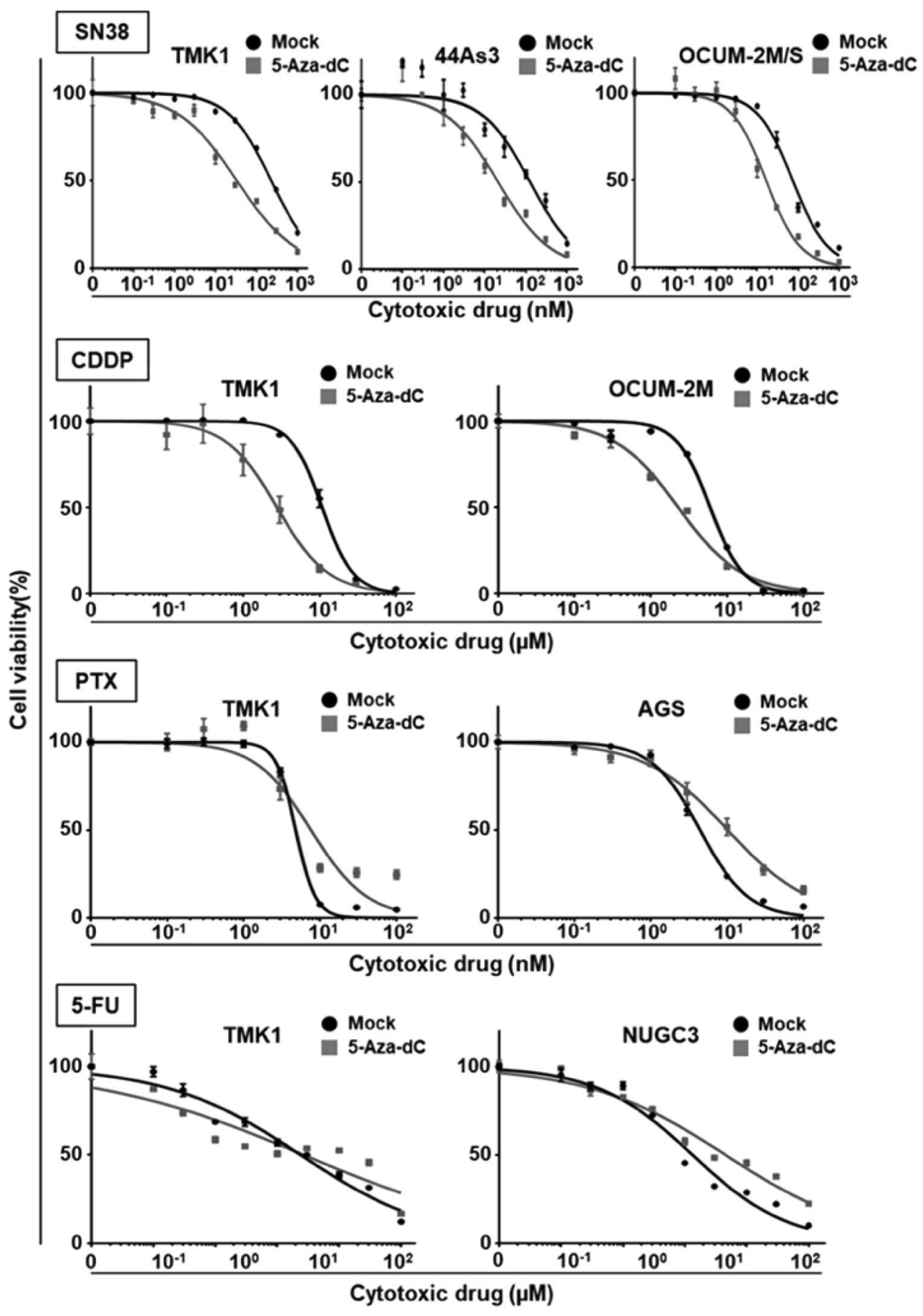

Publisher's Note Springer Nature remains neutral with regard to jurisdictional claims in published maps and institutional affiliations. 